\title{
Effects of Yangzheng Sanjie Decoction-containing serum mediated by microRNA-7 on cell proliferation and apoptosis in gastric cancer
}

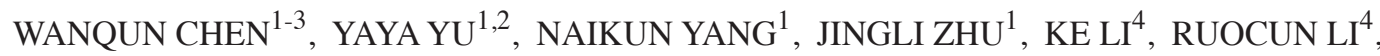 \\ WENQIAO SU ${ }^{4}$, LINA LUO ${ }^{1,2}$, LING HU ${ }^{3}$, GENGXIN $\mathrm{CHEN}^{1,2}$ and HAIXIA DENG ${ }^{5}$
}

\begin{abstract}
${ }^{1}$ Department of Gastroenterology, The Second Affiliated Hospital, Guangzhou University of Chinese Medicine, Guangzhou, Guangdong 510120; ${ }^{2}$ Discipline of Integrated Chinese and Western Medicine, Guangzhou University of Chinese Medicine, Guangzhou, Guangdong 510006; ${ }^{3}$ Institute of Gastroenterology, Guangzhou University of Chinese Medicine, Guangzhou, Guangdong 510405; ${ }^{4}$ Pharmaceutical Research Institute, Hunan Academy of Chinese Medicine, Changsha, Hunan 410013;

${ }^{5}$ Academy of Chinese Medical Sciences, The Second Affiliated Hospital, Guangzhou University of
\end{abstract} Chinese Medicine, Guangzhou, Guangdong 510006, P.R. China

Received December 16, 2015; Accepted May 16, 2017

DOI: $10.3892 / \mathrm{ol} .2018 .7757$

\begin{abstract}
Gastric cancer (GC) is one of the most common types of cancer and a leading cause of cancer-associated mortality. MicroRNAs (miRNAs/miRs) are demonstrated to function as oncomiRs or tumor-suppressor-miRs in GC. miR-7 has been identified to be a tumor suppressor of GC by targeting epidermal growth factor receptor (EGFR). In our previous study, Yangzheng Sanjie Decoction (YZSJD), a traditional Chinese formula, was identified to be effective in alleviating the symptoms and even postponing turnover of precancerous lesions. To elucidate the mechanism of YZSJD, the present study evaluated the effects of YZSJD of the GC MKN-45 cell line and investigated the underlying molecular mechanisms using YZSJD-containing serum (YCS). The expression of miR-7 in GC, normal and adjacent tissue samples
\end{abstract}

Correspondence to: Professor Gengxin Chen, Department of Gastroenterology, The Second Affiliated Hospital, Guangzhou University of Chinese Medicine, 111 Dade Road, Guangzhou, Guangdong 510120, P.R. China

E-mail: chengxlab@126.com

Dr Haixia Deng, Academy of Chinese Medical Sciences, The Second Affiliated Hospital, Guangzhou University of Chinese Medicine, 55 West Inner Ring Road, Guangzhou, Guangdong 510006, P.R. China E-mail: hxd6870@126.com

Abbreviations: GC, gastric cancer; YZSJD, Yangzheng Sanjie Decoction; YCS, YZSJD-containing serum; EGFR, epidermal growth factor receptor; miRNAs, microRNAs; miR-7, microRNA-7; HPLC, high performance liquid chromatography; FBS, fetal bovine serum; CCK-8, Cell Counting kit-8; RT-qPCR, reverse transcription-quantitative polymerase chain reaction; H\&E, hematoxylin and eosin; ISH, in situ hybridization

Key words: Yangzheng Sanjie Decoction, microRNA-7, gastric cancer, epidermal growth factor receptor, MKN-45 cells was examined. The results demonstrated that YCS inhibited proliferation by inducing cell cycle arrest at the $\mathrm{S}$ phase, and significantly induced apoptosis compared with the control group. miR-7 was significantly downregulated in GC tissues compared with the matched ones. Using reverse transcription-quantitative polymerase chain reaction and western blot analysis, the expression of miR-7 was inversely associated with EGFR. This indicates that YCS inhibits proliferation and induces apoptosis of GC cells mediated by miR-7 targeting EGFR, which may be one of the mechanisms whereby YZSJD exerts its effects on GC.

\section{Introduction}

According to the global cancer statistics, an estimated 951,600 new gastric cancer (GC) cases and 723,100 GC-associated mortalities occurred in 2012 (1). Although the incidence and mortality rates of GC have decreased steadily, GC among males remains the third most frequently diagnosed cancer and leading cause of cancer-associated mortality, particularly in Eastern Asia (1). As is known, the mainstay of diagnosis is to confirm the GC lesion histopathologically, while $2 / 3$ patients diagnosed in this way exhibit poor survival, thus prevention and individual treatment are considered the best options to reduce mortality rates (2). Therefore, exploring the specific diagnostic, therapeutic or prognostic biomarkers for $\mathrm{GC}$ remain the key areas of study. It has been revealed that intestinal gastric adenocarcinoma-genesis is due to the multistep process of genetic and epigenetic abnormalities, while diffuse gastric adenocarcinoma occurs predominantly as a result of gene mutations (3).

MicroRNAs (miRNAs/miRs) are small non-coding, single-stranded RNAs that modulate $\sim 30 \%$ of protein translation through binding to target mRNAs (4). Numerous studies have shed light on the role of miRNAs, which are involved in the dysregulation of oncogenes or tumor suppressor genes for cancer (5-7). For GC, it has been revealed that miRNAs 
function as oncomiRs or tumor-suppressor-miRs in the pathogenesis and progression of carcinogenesis (8), or as diagnostic, therapeutic and, prognostic biomarkers (9) involved in cell proliferation, epithelial-mesenchymal transition, apoptosis, migration, invasion and metastasis (10-12).

miR-7, an evolutionarily-conserved miRNA, has been demonstrated as a tumor suppressor in breast cancer, ovarian cancer and glioblastoma, and epidermal growth factor receptor (EGFR) has been identified as its direct target (13-15). Similarly, several studies reported that the expression of miR-7 in the tissue of GC and gastritis is lower than normal tissues, which inhibits metastasis and invasion by targeting EGFR or insulin-like growth factor-1 receptor, and has emerged as a potential therapeutic method for GC (16-18).

It has been revealed that certain Chinese formulae, including Jianpi herbs and Weichang'an induce apoptosis of GC cells $(19,20)$. Yangzheng Xiaoji, used in the treatment of several solid tumors, including GC, has been shown to inhibit cell adhesion, migration and tube formation mediated by the focal adhesion kinase pathway (21).

Yangzheng Sanjie Decoction (YZSJD), a traditional Chinese formula, is composed of Astragali Radix, Scutellariae Barbatae Herba, Arisaematis Rhizoma Preparatum, Citri Sarcodactylis Fructus, Cremastrae Pseudobulbus and Curcumae Longae Rhizoma (22). In clinical practice, it was revealed that YZSJD is effective to alleviate the symptoms, postpone or even reverse the precancerous lesions (intestinal metaplasia or dysplasia) of GC $(23,24)$. The present study explored the mechanism mediated by YZSJD on GC with the method of decoction-containing serum, which has been revealed to be an excellent method for exploring the molecular mechanism of Chinese formulae (25), and it was hypothesized that miR-7 regulates proliferation and apoptosis by targeting EGFR.

\section{Materials and methods}

Tissue samples. Primary GC samples, matched adjacent tumor samples and normal tissue samples were obtained from 11 patients (mean age, 59.2 years old; range, 34-69 years old) with GC undergoing radical gastrectomy at the Department of Surgery, The First Affiliated Hospital, Guangzhou University of Chinese Medicine (Guangzhou, China) between May 2014 and December 2014. The experiments were approved by the Ethics Committee of The First Affiliated Hospital of Guangzhou University of Chinese Medicine. None of the patients received preoperative treatment. Tumor tissue, normal tissue $>3 \mathrm{~cm}$ away from tumors and adjacent tumor tissue between the tumor and normal tissues were collected following gastric resection surgery. Written informed consent was obtained from all patients included and confirmed by pathological examination, and histological typing was performed using Lauren's classification (26). The tumor-node-metastasis classification definitions were according to the Japanese Gastric Cancer Association (27). The clinical parameters are listed in Table I.

Preparation for the medicine. YZSJD, the components of which were obtained from Kangmei Pharmaceutical Co., Ltd. (Guangdong, China) and identified by two pharmacological experts, was prepared as described by Ren et al (28). In order
Table I. Clinicopathologcal parameters of patients with gastric cancer.

\begin{tabular}{lc} 
Clinicopathological parameters & Patients, $\mathrm{n}$ \\
\hline Age, years & \\
$\leq 59$ & 6 \\
>59 & 5 \\
Sex & \\
Male & 8 \\
Female & 3 \\
Location & \\
Middle proximal & 4 \\
Distal & 7 \\
Histological type & \\
Intestinal & 9 \\
Diffuse & 2 \\
Depth of invasion & \\
T1, T2 & 10 \\
T3, T4 & 1 \\
Lymph node metastasis & \\
No & 6 \\
Yes & \\
Differentiation & 5 \\
High & \\
Moderate/low & \\
TNM stage & \\
I, II & \\
III, IV & 2 \\
\hline
\end{tabular}

TNM, tumor-node-metastasis.

to establish the quality standards of the compounds of YZSJD, the preparation of crude extracts and subsequent high-performance liquid chromatography (HPLC) experiments were performed more than three times, as previously described (29).

Preparation of YZSJD-containing serum (YCS). In accordance with the Committee on Ethics of Animal Experiment of The Second Affiliated Hospital of Guangzhou University of Chinese Medicine, 40 8-week old Sprague Dawley male rats, weighing $250 \pm 20 \mathrm{~g}$, were purchased from the Animal Experiment Center of Southern Medical University (Guangzhou, China). The rats were acclimatized to laboratory conditions $\left(23^{\circ} \mathrm{C}, 12 / 12 \mathrm{~h}\right.$ light/dark, $50 \%$ humidity, ad libitum access to food and water) and were divided into two groups and treated with YZSJD or saline, respectively. The YZSJD was dissolved into fluid with sterile water, and an equivalent of the human dose was calculated for the rats, in accordance with the Methodology on Chinese Medicinal Pharmacology (30). The two groups of rats then continuously received intragastric administration of $9 \mathrm{~g} / \mathrm{kg}$ YZSJD or saline daily for 7 days. On the last day of gavage, all the rats were anesthetized with diethyl ether for $1 \mathrm{~h}$ and blood was drawn from the abdominal aorta. Subsequent to remaining still for $\geq 1 \mathrm{~h}$, the blood was centrifuged (at 2,000 x g, $15 \mathrm{~min}$ ) at room temperature, then the serum was collected. The serum from all animals of the same 
group was pooled and inactivated at $56^{\circ} \mathrm{C}$ for $30 \mathrm{~min}$, prior to filtration using a $0.22-\mu \mathrm{m}$ pore filter membrane. Serum was then stored at $-20^{\circ} \mathrm{C}$ until use. Further details of the protocol are available in two previously published studies $(22,31)$.

Cell culture. The human gastric MKN-45 cell line was obtained from the Type Culture Collection of the Chinese Academy of Science (Shanghai, China) and maintained in RPMI-1640 medium (HyClone; GE Healthcare Life Sciences, Little Chalfont, UK), supplemented with $10 \%$ fetal bovine serum (FBS; HyClone; GE Healthcare Life Sciences). Cell culture was conducted at $37^{\circ} \mathrm{C}$ in a humidified $5 \% \mathrm{CO}_{2}$ incubator.

Experimental groups. The MKN-45 cells were divided into two groups as follows: The Chinese medicine (CM) group, treated with high-dose YZSJD drug-containing serum $(20 \%$ added to serum-free RPMI-1640 medium), intermediate-dose YZSJD drug-containing serum $(15 \%)$ or low-dose YZSJD drug-containing serum (10\%), and the control group treated with a matched concentration of YCS treated with saline.

Cell proliferation assay. Cell proliferation was measured with a colorimetric water-soluble tetrazolium salt assay using Cell Counting kit-8 (CCK-8; Beyotime Institute of Biotechnology) and plate cloning according to the manufacturer's protocols. MKN-45 cells in the logarithmic phase were seeded onto 96-well plates $\left(8 \times 10^{3}\right.$ cells, $100 \mu \mathrm{l} /$ well). Following incubation for $24 \mathrm{~h}$ at $37^{\circ} \mathrm{C}$, the culture medium was removed and washed with $\mathrm{PBS}$, and the CM groups were treated with three different concentrations of YCS in FBS-free RPMI-1640 $(10,15$ and $20 \%)$ in order to identify the most appropriate concentration. Similarly, the control was treated at the matched concentration with FBS-containing medium. At $48 \mathrm{~h}$ post-incubation, the optical density (OD) value of each well was measured at $450 \mathrm{~nm}$ using a VICTOR X5 Multimode plate reader (PerkinElmer, Inc., Waltham, MA, USA).

With the most appropriate concentration of YCS (10\%), the cells were resuspended in serum-free RPMI-1640 medium, and $5 \times 10^{2}$ cells were seeded on a 6 -well plate. YCS was then uniformly dropped into the wells of the CM group. Simultaneously, the same amount of FBS was dropped into the wells of the control group. Cells were then incubated for 12 days at $37^{\circ} \mathrm{C}$, without agitation for the first 4 days. Subsequently, the medium was removed and cells were washed with PBS. Cells were fixed in $100 \%$ methanol for $15 \mathrm{~min}$ at room temperature and stained with $0.1 \%$ crystal violet for $15 \mathrm{~min}$ at room temperature. Colonies containing at least 50 cells were counted with a light microscope at magnification, x100 (Olympus Corporation, Tokyo, Japan).

Cell cycle and apoptosis analysis. Randomized MKN-45 $\left(5 \times 10^{5}\right)$ cells were seeded onto 6 -well plates. The same concentration of serum-containing medium (10\%) was used, and cells were incubated for the same length of time as the cell proliferation procedure. The medium of each well was then collected in the specific tube and washed with PBS. Using trypsin (Gibco; Thermo Fisher Scientific, Inc.) without EDTA, the cells were digested and collected in the tube. Following centrifugation twice (168 x g for $5 \mathrm{~min}$ at room temperature) and washing with PBS, the staining procedure was performed in accordance with the fluorescein isothiocyanate Annexin V apoptosis detection kit I (BD Biosciences, Franklin Lakes, NJ, USA). The specimens were incubated at room temperature for 15 min and measured using a Cytomics ${ }^{\mathrm{TM}} 500$ flow cytometer (Beckman Coulter, Inc., Brea, CA, USA). While the cell cycle kit was purchased from Beyotime Institute of Biotechnology (Haimen, China), all the procedures were performed according to the manufacturers' protocols.

For the morphology of different group of cells, Hoechst 33258 (Beyotime Institute of Biotechnology) was used to stain the cells for $5 \mathrm{~min}$ at room temperature, and the cells were then observed at magnification, x200 under a fluorescence microscope (Olympus Corporation, Tokyo, Japan) and images were captured.

Reverse transcription-quantitative polymerase chain reaction $(R T-q P C R)$. Total RNA was extracted from cells with TRIzol reagent (Invitrogen; Thermo Fisher Scientific, Inc.). The PCR primers were as follows: miR-7 forward, 5'-CTGTTACTATGG TAGCGACACTG-3' and reverse, 5'-CACACTGGAGGATTA CATTCCC-3'; U6 forward, 5'-CTCGCTTCGGCAGCACA-3' and reverse, 5'-AACGCTTCACGAATTTGCGT-3'. Reverse transcription was performed with the Bulge-Loop ${ }^{\mathrm{TM}}$ miRNA RT-qPCR Primer (Guangzhou RiboBio Co., Ltd., Guangzhou, China) and the PrimeScript ${ }^{\mathrm{TM}} \mathrm{RT}$ reagent (Takara Bio, Inc., Otsu, Japan) kits, according to the manufacturers' protocols. RT-qPCR was performed on Axygen 96-well reaction plates with the Applied Biosystems 7500 Real-time PCR System following and the SYBR Premix Ex Taq kit (Takara Bio, Inc.), according to the manufacturer's protocol. The thermocycling conditions were as previously described (22). According to the instructions of the Bulge-Loop miRNA RT-qPCR Primer, a U6 small nuclear RNA was used as the internal reference. Data were analyzed using the $2^{-\Delta \Delta \mathrm{Cq}}$ method (32). The experiments were repeated at least three times.

Western blot analysis. Protein was extracted from the MKN-45 cell line using radioimmunoprecipitation assay buffer containing a protease inhibitor cocktail (Beyotime Institute of Biotechnology) according to the manufacturer's protocol. The protein concentration of each sample was quantified using a bicinchoninic acid assay (Beyotime Institute of Biotechnology, Haimen, China). Subsequently, equal amounts of proteins $(60 \mu \mathrm{g})$ were separated by electrophoresis on $10 \%$ polyacrylamide gels and transferred onto $0.2 \mu \mathrm{m}$ polyvinylidene difluoride membranes. The membranes were blocked with 5\% milk in Tris-buffered saline and $0.2 \%$ Tween at room temperature for $1 \mathrm{~h}$, prior to being incubated overnight at $4^{\circ} \mathrm{C}$ with specific primary antibodies against the following: $\beta$-actin (dilution, 1:250; cat. no. BM0627; Boster Biological Technology, Wuhan, China) and EGFR (dilution, 1:1,000; cat. no. 4267S; CST Biological Reagents Co., Ltd., Shanghai, China). The blots were washed three times with Tris-buffered saline and $0.2 \%$ Tween (TBST) and incubated with a goat anti-rabbit secondary antibody IgG-HRP (dilution, 1:1,000; cat. no. BA1054; Boster Biological Technology) for $1 \mathrm{~h}$ at room temperature, prior to being washed with TBST ( 3 times, each for $10 \mathrm{~min})$. The expression signals were detected using BeyoECL Plus (Beyotime Institute of Biotechnology) according to the manufacturer's protocols. Quantification of the bands was 

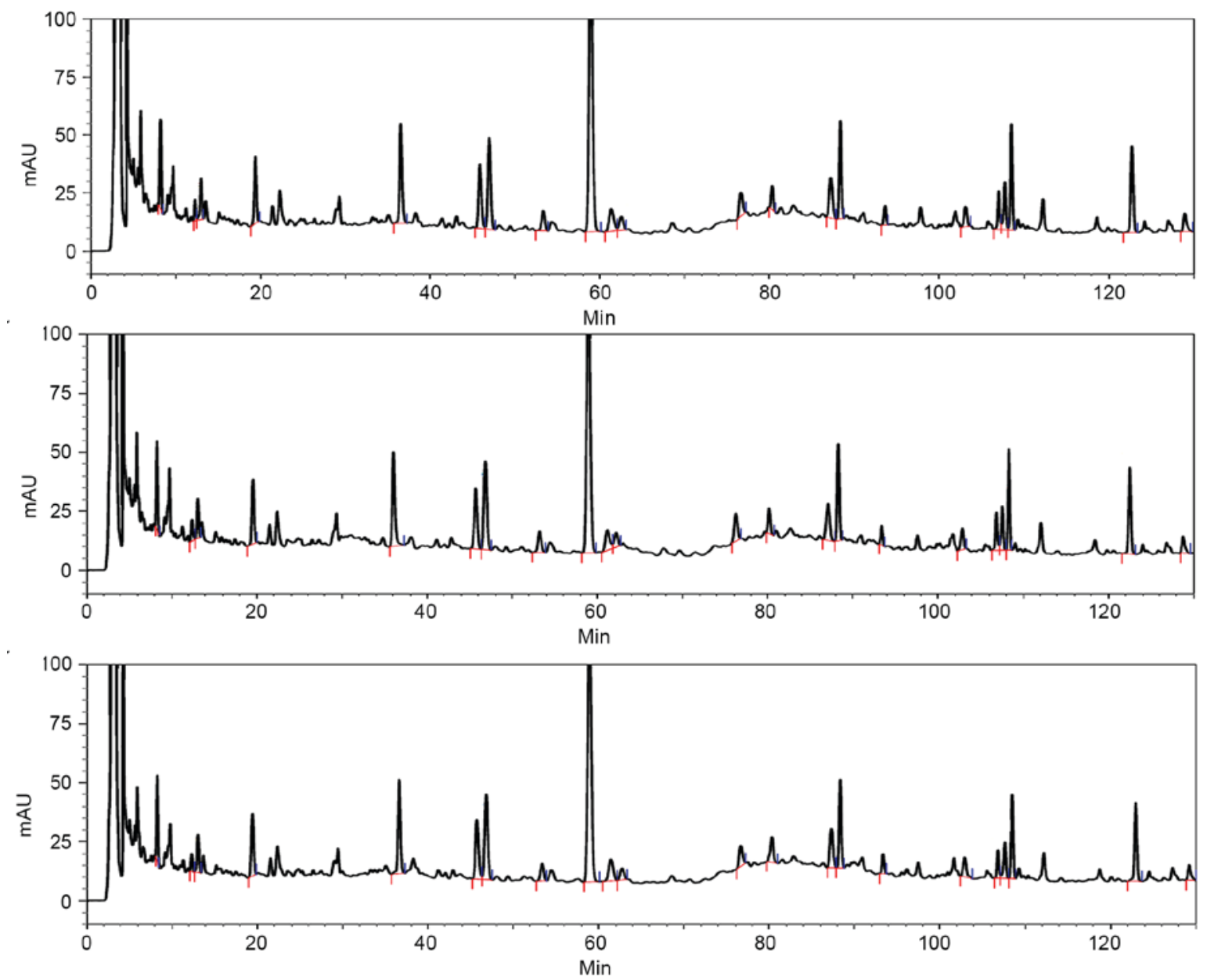

Figure 1. HPLC chromatograms of Yangzheng Sanjie Decoction. HPLC experiments were performed more than three times, and the results demonstrated the stability of the extracts and the preparation technology. HPLC, high phase liquid chromatography.

performed using Image Lab software (version 4.1; Bio-Rad Laboratories, Inc., Hercules, CA, USA) and the protein expression levels were normalized to the levels of $\beta$-actin.

Hematoxylinandeosin $(H \& E)$ staining and in situ hybridization $(I S H)$. All the tissues obtained were fixed with $4 \%$ paraformaldehyde for $15 \mathrm{~min}$ at room temperature, paraffin-embedded and sectioned. To ascertain the actual histology of every sample, H\&E staining was performed routinely. The samples were then observed with a light microscope (Olympus Corporation, Tokyo, Japan) at magnification, x200 by two pathology experts blindly. ISH was performed as follows: Digoxigenin-labeled locked nucleic acid modified probes for miR-7 were obtained from Exiqon A/S, Vedbaek, Denmark. Briefly, 4- $\mu \mathrm{m}$ sections of the tissue microarray blocks were placed in a heater at $55^{\circ} \mathrm{C}$ overnight. Sections were deparaffinized with $2 \mathrm{X}$ xylene ( 5 min), rehydrated with ethanol (100, 50 and $25 \%$ for $5 \mathrm{~min}$ each), and treated with diethyl pyrocarbonate water for $1 \mathrm{~min}$ at room temperature. Protease treatment was performed with pepsin solution $(1.3 \mathrm{mg} / \mathrm{ml})$ at $37^{\circ} \mathrm{C}$ for $50 \mathrm{~min}$. Following a post-fixation step in $4 \%$ paraformaldehyde at room temperature for $10 \mathrm{~min}$, hybridization of the locked nucleic acid probe was performed at $52^{\circ} \mathrm{C}$ for $5 \mathrm{~min}$ followed by $37^{\circ} \mathrm{C}$ overnight. A low-stringency post-hybridization wash was performed at $4^{\circ} \mathrm{C}$ in saline sodium citrate with $2 \%$ bovine serum albumin for $5 \mathrm{~min}$, followed by incubation with anti-digoxigenin primary antibody (dilution, 1:1,000; cat. no. ab6212; Abcam, Cambridge, UK) at $37^{\circ} \mathrm{C}$ for $30 \mathrm{~min}$. The slides were counterstained with $1 \%$ hematoxylin for $1 \mathrm{~min}$ at room temperature to visualize the nuclei prior to cover glass mounting. The mean proportion of stained cells in every specimen was screened quantitatively and scored as previously described (16).

Statistical analysis. The results are presented as the mean \pm standard error of the mean. Statistical analyses were performed with Stata 12.1 (StataCorp, LLC, College Station, TX, USA). One-way analysis of variance followed by Dunnett's test, or an unpaired Student's t-test was applied for statistical analysis. For comparison of paired tissues, a paired Student's t-test was used to determine the statistical significance. A two-tailed value of $\mathrm{P}<0.05$ was considered to indicate a statistically significant difference.

\section{Results}

HPLC. Fig. 1 presents the stability of the extracts and the preparation technology of the compound of YZSJD.

YCS inhibits GC cell proliferation. The effect of YCS on the viability of MKN-45 cells was determined using a CCK-8 
A

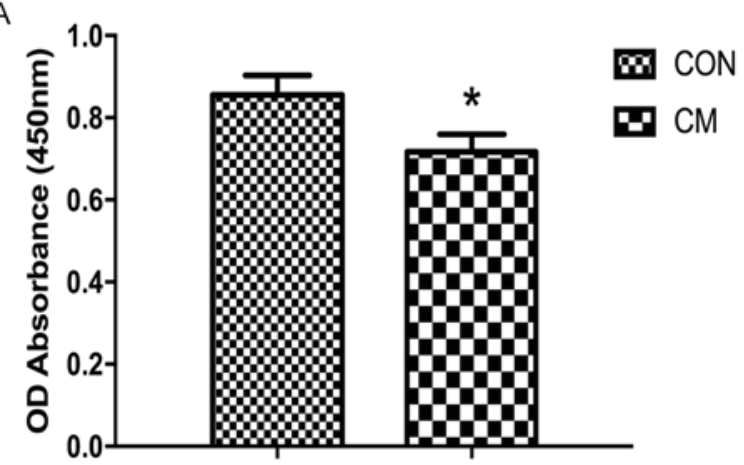

B

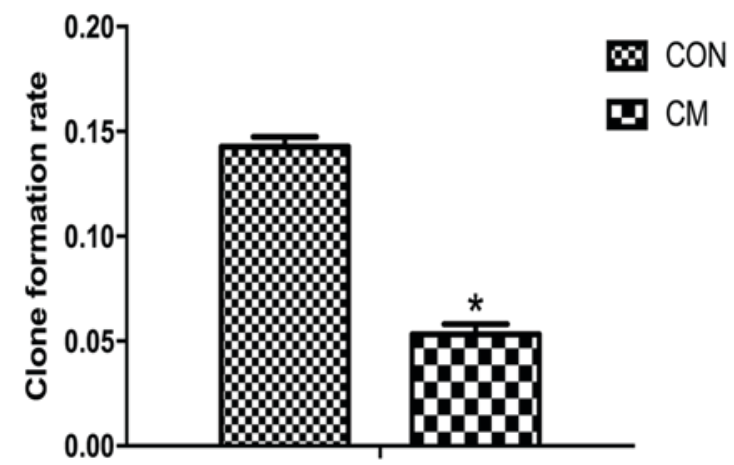

C

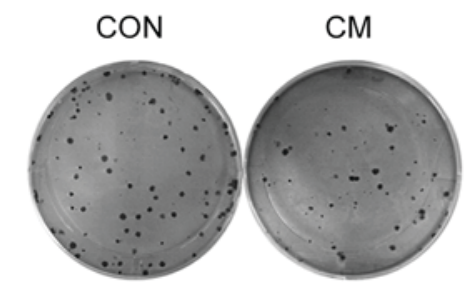

Figure 2. Effects of Yangzheng Sanjie Decoction-containing serum on cell proliferation. (A) Optical density absorbance in the CM group was decreased compared with the control group. (B) Histogram of clone formation rate for the $\mathrm{CM}$ and control groups. ${ }^{*} \mathrm{P}<0.05$. (C) Representative image of cell clone formation. CM, Chinese medicine; Con, control; miR-7, microRNA-7.

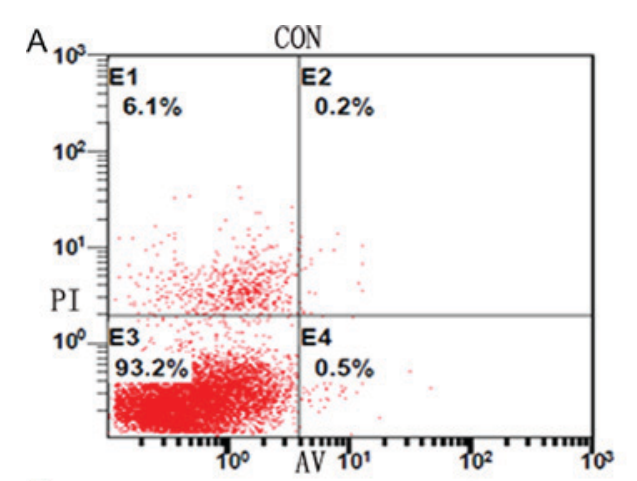

C

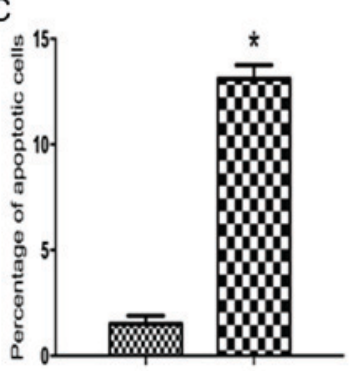

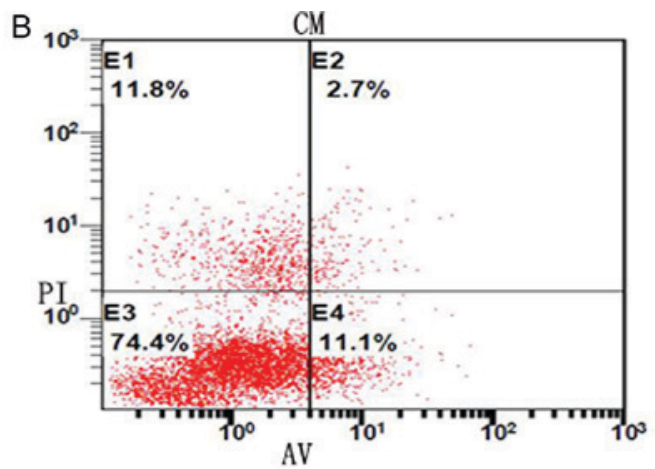

$\mathrm{AV}$
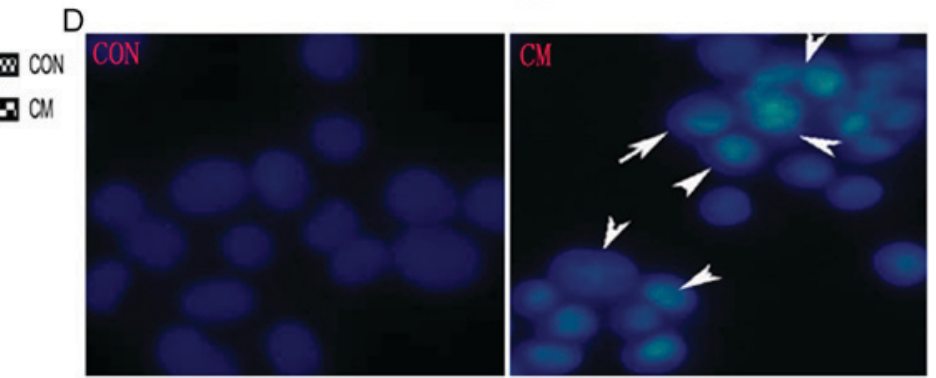

Figure 3. Effects of YCS on cell apoptosis. (A) Cell apoptosis in the CM group; cell staining positive for Annexin-V-fluorescein isothiocyanate and negative for PI at $48 \mathrm{~h}$ post-YCS-treatment were considered to have undergone apoptosis. (B) Cell apoptosis in the control group. (C) Histograms of the percentage of apoptotic cells. "P<0.05. (D) Morphological characteristics of apoptotic cells in the CM group (arrows). YCS, Yangzheng Sanjie Decoction-containing serum; PI, propidium iodide; CM, Chinese medicine; Con, control; AV, Annexin V.

assay. YCS (10\%) and FBS-containing media were added to the CM and control groups, respectively. Using CCK-8 reagent, the OD in different groups was examined and it was revealed that the cells in the CM group proliferated more slowly compared with those of the control group. Proliferation of MKN-45 cells in the CM group was downregulated compared with the control group $(\mathrm{P}<0.05$; Fig. 2A). In addition, YCS inhibited colony formation ability of MKN-45 cells compared with the control group (Fig. 2B and $\mathrm{C}$ ).

YCS induces GC cell apoptosis. To elucidate whether YCS inhibits the growth of GC cells by inducing apoptosis, the exposure of phosphatidylserine on the cell surface was examined by Annexin V/propidium iodide staining, followed by fluorescence-activated cell sorting analysis. The percentage 

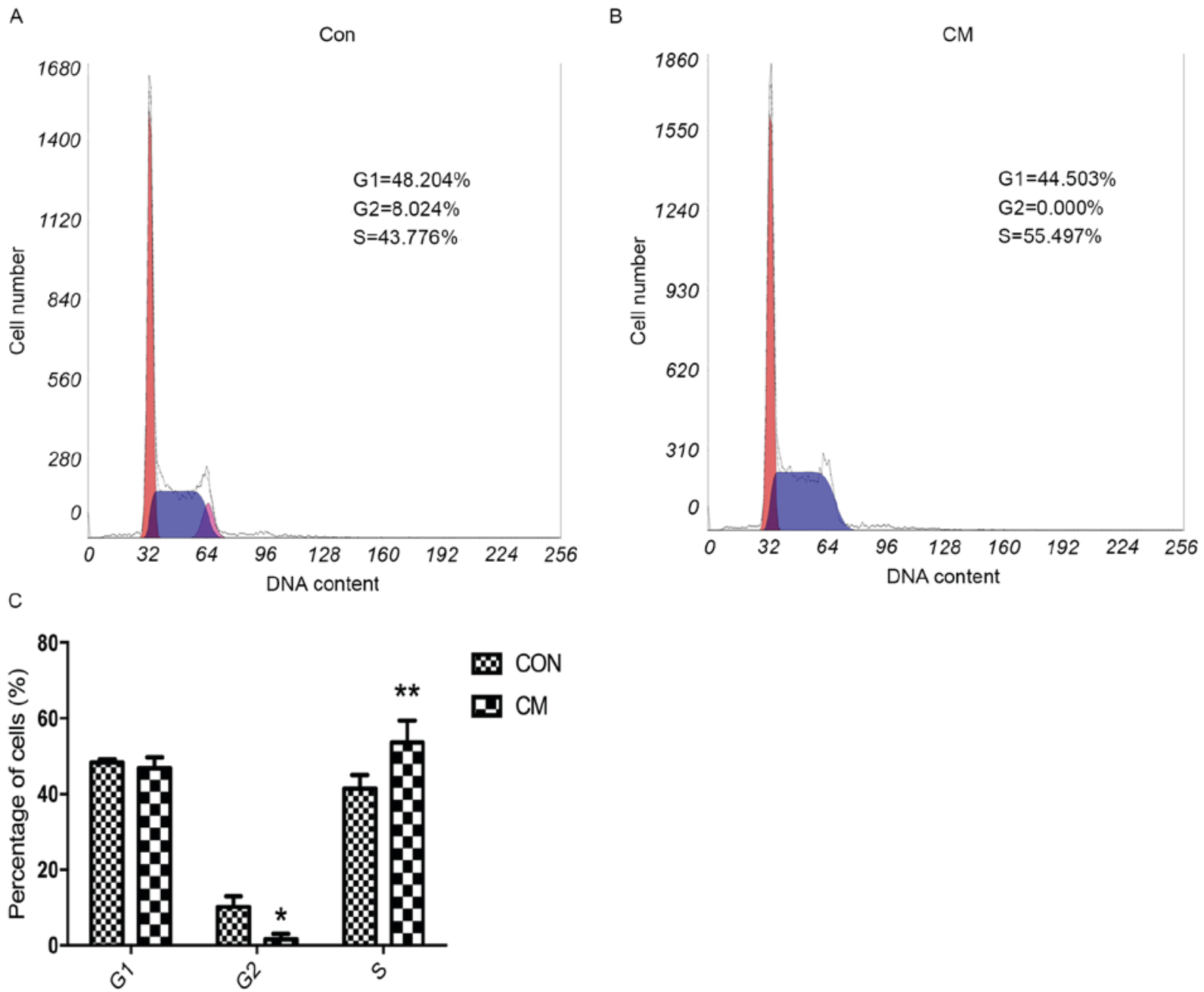

Figure 4. Effects of YCS on cell cycle distribution. (A) Proportion of cells in various phases of the cell cycle in the (A) Con group and (B) CM group. (C) Cell cycle analysis of the CM and Con groups. "P<0.05; ${ }^{* *} \mathrm{P}<0.01$. YCS, Yangzheng Sanjie Decoction-containing serum; CM, Chinese medicine; Con, control.

A

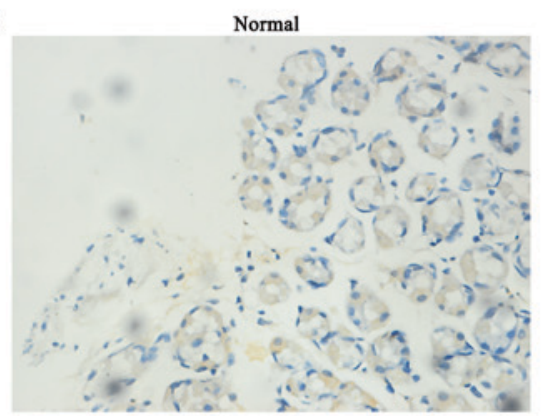

B

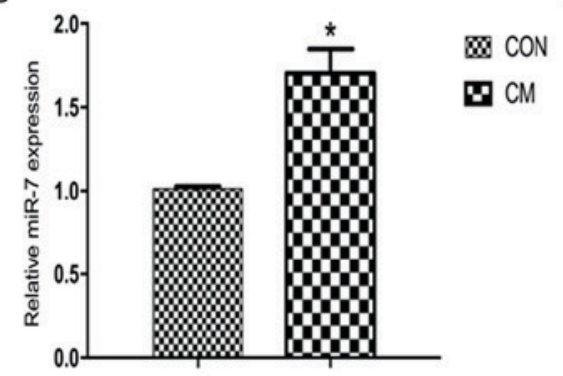

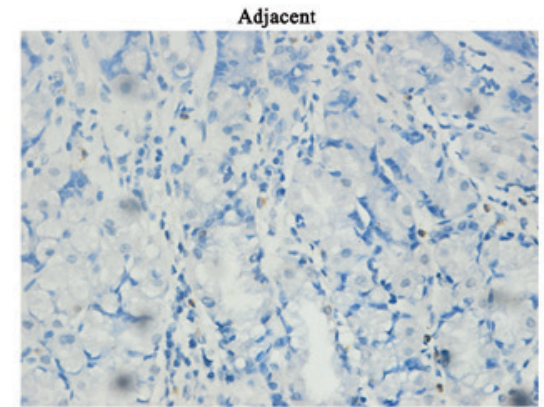

C

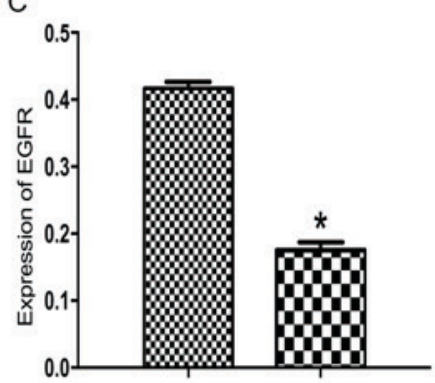

GC

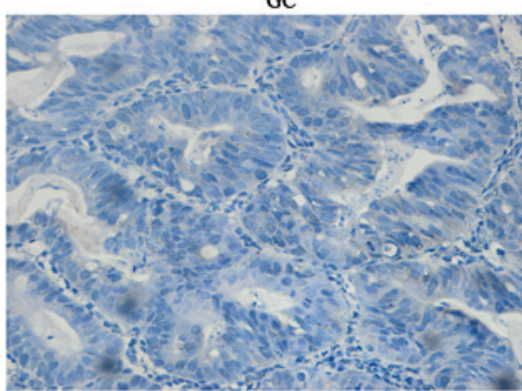

D m CON

$\mathbf{W} \mathrm{CM}$

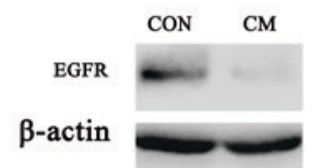

Figure 5. Expression of miR-7 and EGFR. (A) Hematoxylin staining indicating the expression of miR-7 by in situ hybridization (magnification, x400). (B) Expression of miR-7 for reverse transcription-quantitative polymerase chain reaction. U6 was used as the internal control. (C) Western blot analysis of EGFR. (D) Representative image of EGFR for western blot analysis. $\beta$-actin was used as the internal control. "P<0.05. miR-7, microRNA-7; EGFR, epidermal growth factor receptor; CM, Chinese medicine; Con, control; GC, gastric cancer. 
Table II. microRNA-7 expression of in situ hybridization.

\begin{tabular}{lcccc}
\hline Tissue & Negative (-) & Weak $(+)$ & Moderate $(++)$ & Strong $(+++)$ \\
\hline Normal & 0 & 4 & 1 & 6 \\
Adjacent & 1 & 7 & 3 & 0 \\
Gastric cancer & 9 & 2 & 0 & 0 \\
\hline
\end{tabular}

of cells in early or late apoptosis was calculated. As presented in Fig. 3A and B, a significantly higher percentage of apoptotic cells was observed in the CM group compared with the control group, indicating that YCS induced apoptosis in Fig. 3C $(\mathrm{P}<0.05)$.

For additional assessment of apoptosis induced by YCS, the morphological characteristics of apoptosis were screened. GC cells treated with YCS for $48 \mathrm{~h}$ were stained with Hoechst 33258 and observed by fluorescence microscopy. As presented in Fig. 3D, apoptotic cells exhibited typical changes in the CM group, including condensed and fragmented nuclei, whereas control cells exhibited an even distribution of the stain and round homogeneous nuclei.

$Y C S$ induces cell cycle arrest in the S phase. Cell cycle analysis was performed to determine the effect of YCS in cell cycle alteration. Results demonstrated that, compared with the control group, the percentage of MKN-45 cells in the CM group was significantly increased at the $S$ phase and decreased at the $G_{2}$ phase (Fig. 4). These results indicated that YCS inhibited GC cell proliferation by inducing cell cycle arrest at the $\mathrm{S}$ phase.

Expression of miR-7 is downregulated in GC clinical samples. According to the results of H\&E staining, every GC sample had a corresponding matched normal tissue sample. To assess the expression of miR-7 in different tissues, miR-7 expression was screened in 11 GC clinical samples using ISH. It was revealed that GC tissues had a markedly low expression level of miR-7 compared with matched normal tissues and adjacent tissues, indicating a decreasing trend among the three of them, as presented in Fig. 5A and Table II. Statistical significance was observed among the GC, adjacent and normal tissues $(\mathrm{P}<0.05)$.

YCS mediates the function of miR-7 by targeting EGFR. The results of the qPCR assay demonstrated that YCS treatment significantly upregulated the expression of miR-7 in MKN-45 cells compared with that in control cells $(\mathrm{P}<0.05$; Fig. 5B). By contrast, the protein level of EGFR in the CM group was significantly lower compared with that of the control group $(\mathrm{P}<0.05$; Fig. 5C and D). Consistently, using ISH, the expression of miR-7 was markedly downregulated in GC tissue $(\mathrm{P}<0.05$; Fig. 5A), indicating that miR-7 may serve as a tumor suppressor targeting EGFR. Consequently, these results suggest that YCM inhibits proliferation and induces apoptosis mediated by miR-7 targeting EGFR.

\section{Discussion}

To the best of our knowledge, surgical resection remains the only cure for GC, which is possible in only $25-30 \%$ of cases, suggesting that GC is one of the malignancies with the highest mortality rate (33). With a medium follow-up time of $>10$ years, overall survival and relapse-free survival rates have continually benefited from postoperative radiochemotherapy (34). However, a number of anticancer agents contain intrinsic and potent cytotoxicity to normal cells, which limits their long-term use and their therapeutic effectiveness, emphasizing the need to develop novel anticancer drugs (35).

Traditional Chinese medicines (TCM), which have been used for thousands of years, have relatively higher safety compared with typical anticancer agents and have attracted great interest for cancer treatment $(36,37)$. According to the medicine compatibility, TCMs consist of numerous natural products, including principal, assistant, adjuvant and dispatcher herbs in specific formula, which have the function of increased therapeutic effect, synergistic action, targeting specific lesion, treatment of complex diseases, and relieving side effects (38).

Correa's multi-stage cascade of gastric oncogenesis is proposed to be triggered by Helicobacter pylori, progression of atrophic gastritis, intestinal metaplasia, dysplasia and GC (3), suggesting its complex pathology, and multifactor crosstalk effect. Similarly, TCM also considers that GC is a sophisticated disease with multiple factors working together, resulting from internal deficiency and relative pathogenic factors, including toxins, phlegm and blood stagnation (39). YZSJD is a formula contributing to regulate the disorder of human homeostasis, in which the ethanol extract of Scutellaria Barbata D. Don has been revealed to suppress the activation of several colorectal cancer-associated pathways (39). Cremastra appendiculata tuber, the other type of Cremastrae Pseudobulbus is reported to decrease the metastatic nodules and relieve symptoms of metastatic bladder cancer in the lungs with several herbs (40). In addition, Yangzheng Xiaoji, a similar formula in which the exact composition is unknown, has been demonstrated to inhibit angiogenesis, thus exhibiting an anticancer effect (21). By clinical observation, it was demonstrated that YZSJD exhibits improved therapeutic outcome for precancerous gastric lesions (atrophic gastritis) compared with the control group, and with decreased protein expression of EGF and EGFR in the gastric mucosa $(22,24)$. However, whether YZSJD has an effect on GC, and the exact mechanism of this, remains uncovered.

By contrast, a large number of studies demonstrated that miRNAs perform a crucial role in gastric carcinogenesis and serve as useful clinical biomarkers (41-43). Furthermore, the expression of miR-7 has been demonstrated to decrease in GC tissue compared with normal tissue, suggesting that it inhibits proliferation, metastasis and invasion by targeting EGFR (17). Therefore, two tissue samples adjacent to the tumor and one normal tissue sample were used to explore whether miR-7 is 
dysregulated in the precancerous lesion. The present data supported the hypothesis and EGFR was proposed as the target.

In the present in vitro study, YCS was prepared according to the previously published serum pharmacology method which is stable and dependable (28,31). Proliferation and apoptosis were compared between the CM and control groups, and the results revealed that YCS inhibits proliferation, and induces apoptosis and cell cycle arrest. Simultaneously, RT-qPCR revealed that the expression of miR-7 was significantly upregulated in the YCS-treated cells compared with control cells, whereas western blot analysis revealed that the expression of EGFR was significantly downregulated (Fig. 5C), consistent with the results in GC, adjacent and normal tissues (Table II). On the basis of these results, it was concluded that YCS inhibits proliferation and induces apoptosis of GC cells, which is mediated by miR-7 targeting EGFR.

\section{Acknowledgements}

The present study was supported by the National Nature Science Foundation of China (grant nos. 81273735 and 81373563), the Science and Technology Planning Project of Guangdong Province, China (grant nos. 2016A020215135 and 2013B021800169) and the Traditional Chinese Medicine Science and Technology Research Projects of Guangdong Provincial Hospital of Chinese Medicine, China (grant nos. YN2014ZH05 and YN2016ML06).

\section{References}

1. Torre LA, Bray F, Siegel RL, Ferlay J, Lortet-Tieulent J and Jemal A: Global cancer statistics, 2012. CA Cancer J Clin 65: $87-108,2015$

2. Hartgrink HH, Jansen EP, van Grieken NC and van de Velde CJ: Gastric cancer. Lancet 374: 477-490, 2009.

3. Yakirevich E and Resnick MB: Pathology of gastric cancer and its precursor lesions. Gastroenterol Clin North Am 42: 261-284, 2013.

4. Filipowicz W, Bhattacharyya SN and Sonenberg N: Mechanisms of post-transcriptional regulation by microRNAs: Are the answers in sight? Nat Rev Genet 9: 102-114, 2008.

5. Oom AL, Humphries BA and Yang C: MicroRNAs: Novel players in cancer diagnosis and therapies. Biomed Res Int 2014: 959461, 2014.

6. Lu J, Getz G, Miska EA, Alvarez-Saavedra E, Lamb J, Peck D, Sweet-Cordero A, Ebert BL, Mak RH, Ferrando AA, et al: MicroRNA expression profiles classify human cancers. Nature 435: 834-838, 2005.

7. Tong F, Cao P, Yin Y, Xia S, Lai R and Liu S: MicroRNAs in gastric cancer: From benchtop to bedside. Dig Dis Sci 59: 24-30, 2014.

8. Pan HW, Li SC and Tsai KW: MicroRNA dysregulation in gastric cancer. Curr Pharm Des 19: 1273-1284, 2013.

9. Kim SY, Jeon TY, Choi CI, Kim DH, Kim DH, Kim GH, Ryu DY, Lee BE and Kim HH: Validation of circulating miRNA biomarkers for predicting lymph node metastasis in gastric cancer. J Mol Diagn 15: 661-669, 2013.

10. Bushati N and Cohen SM: microRNA functions. Annu Rev Cell Dev Biol 23: 175-205, 2007.

11. Calin GA and Croce CM: MicroRNA signatures in human cancers. Nat Rev Cancer 6: 857-866, 2006.

12. Croce CM: Causes and consequences of microRNA dysregulation in cancer. Nat Rev Genet 10: 704-714, 2009.

13. Li J, Zheng Y, Sun G and Xiong S: Restoration of miR-7 expression suppresses the growth of Lewis lung cancer cells by modulating epidermal growth factor receptor signaling. Oncol Rep 32: 2511-2516, 2014.

14. Liu Z, Jiang Z, Huang J, Huang S, Li Y, Yu S, Yu S and Liu X: miR-7 inhibits glioblastoma growth by simultaneously interfering with the PI3K/ATK and Raf/MEK/ERK pathways. Int J Oncol 44: 1571-1580, 2014.
15. Masuda M, Miki Y, Hata S, Takagi K, Sakurai M, Ono K, Suzuki K, Yang Y, Abe E, Hirakawa $\mathrm{H}$, et al: An induction of microRNA, miR-7 through estrogen treatment in breast carcinoma. J Transl Med 10 (Suppl 1): S2, 2012.

16. Zhao X, Dou W, He L, Liang S, Tie J, Liu C, Li T, Lu Y, Mo P, Shi Y, et al: MicroRNA-7 functions as an anti-metastatic microRNA in gastric cancer by targeting insulin-like growth factor-1 receptor. Oncogene 32: 1363-1372, 2013.

17. Xie J, Chen M, Zhou J, Mo MS, Zhu LH, Liu YP, Gui QJ, Zhang L and Li GQ: miR-7 inhibits the invasion and metastasis of gastric cancer cells by suppressing epidermal growth factor receptor expression. Oncol Rep 31: 1715-1722, 2014.

18. Kong D, Piao YS, Yamashita S, Oshima H, Oguma K, Fushida S, Fujimura T, Minamoto T, Seno H, Yamada Y, et al: Inflammation-induced repression of tumor suppressor miR-7 in gastric tumor cells. Oncogene 31: 3949-3960, 2012.

19. Zhao AG, Zhao HL, Jin XJ, Yang JK and Tang LD: Effects of Chinese Jianpi herbs on cell apoptosis and related gene expression in human gastric cancer grafted onto nude mice. World J Gastroenterol 8: 792-796, 2002.

20. Zhao AG, Yang JK, You SF, Li T, Zhao HL, Gu Y, Tang LD and Qiu JX: Effects of Chinese herbal recipe Weichang'an in inducing apoptosis and related gene expression in human gastric cancer grafted onto nude mice. Zhong Xi Yi Jie He Xue Bao 5: 287-297, 2007 (In Chinese).

21. Jiang WG, Ye L, Ji K, Frewer N, Ji J and Mason MD: Inhibitory effects of Yangzheng Xiaoji on angiogenesis and the role of the focal adhesion kinase pathway. Int J Oncol 41: 1635-1642, 2012.

22. Deng HX, Yu YY, Zhou AQ, Zhu JL, Luo LN, Chen WQ, $\mathrm{Hu} \mathrm{L}$ and Chen GX: Yangzheng sanjie decoction regulates proliferation and apoptosis of gastric cancer cells by enhancing let-7a expression. World J Gastroenterol 23: 5538-5548, 2017.

23. Chen GX and Li HG: Influences of Yangzheng Sanjie Decoction on EGF, EGFR of gastric mucosa at precancerosis of chronic atrophic gastritis. Guiding J of TCM: 20-22, 2007.

24. Chen GX, Mou T and Li HG: 55 cases of clinic observation for the precancerous lesions (chronic atrophic gastritis) treatment on Yangzheng Sanjie Decoction. Gilin TCM: 17-18, 2007.

25. Iwama H, Amagaya S and Ogihara Y: Effect of shosaikoto, a Japanese and Chinese traditional herbal medicinal mixture, on the mitogenic activity of lipopolysaccharide: A new pharmacological testing method. J Ethnopharmacol 21: 45-53, 1987.

26. Lauren P: The two histological main types of gastric carcinoma: diffuse and so-called intestinal-type carcinoma. An attempt at a histo-clinical classification. Acta Pathol Microbiol Scand 64: 31-49, 1965

27. Japanese Gastric Cancer Association: Japanese classification of gastric carcinoma-2nd English edition. Gastric Cancer 1: 10-24, 1998.

28. Ren M, Zhang J, Wang B, Liu P, Jiang H, Liu G and Yin H: Qindan-capsule inhibits proliferation of adventitial fibroblasts and collagen synthesis. J Ethnopharmacol 129: 53-58, 2010.

29. Zheng F, Wu J, Li X, Tang Q, Yang L, Yang X, Wu W and Hann SS: Chinese herbal medicine Fuzheng Kang-Ai Decoction inhibited lung cancer cell growth through AMPK $\alpha$-mediated induction and interplay of IGFBP1 and FOXO3a. Evid Based Complement Alternat Med 2016: 5060757, 2016.

30. Iwama H, Amagaya S and Ogihara Y: Effect of shosaikoto, a Japanese and Chinese traditional herbal medicinal mixture, on the mitogenic activity of lipopolysaccharide: A new pharmacological testing method. J Ethnopharmacol 21: 45-53, 1987.

31. Yijing L, Liu H, Yuan C, Wang B, Ren M, Yan L, Wang X and Zhang J: The effects of qindan-capsule-containing serum on the TGF- $\beta 1 /$ ERK signaling pathway, matrix metalloproteinase synthesis and cell function in adventitial fibroblasts. Pharm Biol 51: 712-721, 2013.

32. Livak KJ and Schmittgen TD: Analysis of relative gene expression data using real-time quantitative PCR and the 2(-Delta Delta C(T)) method. Methods 25: 402-408, 2001.

33. Wang J, Yu JC, Kang WM and Ma ZQ: Treatment strategy for early gastric cancer. Surg Oncol 21: 119-123, 2012

34. Smalley SR, Benedetti JK, Haller DG, Hundahl SA, Estes NC, Ajani JA, Gunderson LL, Goldman B, Martenson JA, Jessup JM, et al: Updated analysis of SWOG-directed intergroup study 0116: A phase III trial of adjuvant radiochemotherapy versus observation after curative gastric cancer resection. J Clin Oncol 30: 2327-2333, 2012.

35. Lordick F, Lorenzen S, Yamada Y and Ilson D: Optimal chemotherapy for advanced gastric cancer: Is there a global consensus? Gastric Cancer 17: 213-225, 2014. 
36. Gordaliza M: Natural products as leads to anticancer drugs. Clin Transl Oncol 9: 767-776, 2007.

37. Ji HF, Li XJ and Zhang HY: Natural products and drug discovery. Can thousands of years of ancient medical knowledge lead us to new and powerful drug combinations in the fight against cancer and dementia? EMBO Rep 10: 194-200, 2009.

38. Bian ZX, Xu H, Lu AP, Lee MS and Cheung H: Insights of Chinese medicine syndrome study: From current status to future prospects. Chin J Integr Med 20: 326-331, 2014.

39. Lin J, Chen Y, Cai Q, Wei L, Zhan Y, Shen A, Sferra TJ and Peng J: Scutellaria Barbata D Don inhibits colorectal cancer growth via suppression of multiple signaling pathways. Integr Cancer Ther 13: 240-248, 2014.

40. Lee DH, Kim SS, Seong S, Woo CR and Han JB: A case of metastatic bladder cancer in both lungs treated with korean medicine therapy alone. Case Rep Oncol 7: 534-540, 2014.
41. Wu WK, Lee CW, Cho CH, Fan D, Wu K, Yu J and Sung JJ: MicroRNA dysregulation in gastric cancer: A new player enters the game. Oncogene 29: 5761-5771, 2010.

42. Link A, Kupcinskas J, Wex T and Malfertheiner P: Macro-role of microRNA in gastric cancer. Dig Dis 30: 255-267, 2012.

43. Wu HH, Lin WC and Tsai KW: Advances in molecular biomarkers for gastric cancer: miRNAs as emerging novel cancer markers. Expert Rev Mol Med 16: e1, 2014.

(i) (9) This work is licensed under a Creative Commons Attribution-NonCommercial-NoDerivatives 4.0 International (CC BY-NC-ND 4.0) License. 